\title{
ЗАДАЧА УПРАВЛЕНИЯ ПРОЦЕССОМ КАТАЛИТИЧЕСКОГО РИФОРМИНГА И МЕТОД ЕЕ РЕШЕНИЯ
}

\author{
Матвейкин Валерий Григорьевич', \\ ipu@ahp.tstu.ru
}

\section{Дмитриевский Борис Сергеевич', dmiboris@yandex.ru}

\author{
Кокуев Андрей Геннадьевич², \\ kokuevag@gmail.com
}

\author{
Джамбеков Азамат Матифулаевич², \\ azamat-121@mail.ru \\ 1 Тамбовский государственный технический университет, \\ Россия, 392000, г. Тамбов, ул. Советская, 106. \\ ${ }^{2}$ Астраханский государственный технический университет, \\ Россия, 414056, г. Астрахань, ул. Татищева, 16.
}

Актуальность исследования обусловлена необходимостью обеспечения оптимальных соотношений октанового числа бензина и издержек организации процесса на установке каталитического риформинга мощностью 1 млн т/год по сырью в условиях переменного качества сырья и качества топливного газа. Эффективность производства бензина зависит не только от повышения октанового числа, но и от снижения издержек организации процесса. Существующие системы управления процессом каталитического риформинга не обеспечивают достижения оптимальных соотношений октанового числа бензина и издержек организации процесса. Необходима разработка системы управления процессом каталитического риформинга, обеспечивающей достижение данных соотношений.

Цель: достижение оптимальных соотношений октанового числа бензина и издержек организации процесса на установке каталитического риформинга мощностью 1 млн т/год по сырью в условиях переменного качества сырья и качества топливного газа путем разработки системы управления.

Объект: установка каталитического риформинга мощностью 1 млн т/год по сырью.

Методы исследования основаны на использовании теории нечетких множеств, теории принятия решений в нечетких условиях, вычислительной математики, теории автоматического управления.

Результаты. Поставлена задача управления процессом каталитического риформинга на установке риформинга Л35-11/1000 мощностью 1 млн т/год по сырью. Проведен анализ процесса как объекта управления с выделением его особенностей. Описан набор входных и выходных параметров объекта управления. Представлено выражение для определения обобщенного критерия оптимальности. Разработана система управления процессом каталитического риформинга. Реализация предлагаемой системы обеспечивает достижение оптимальных соотношений октанового числа бензина и производственных затрат.

\section{Ключевые слова:}

Каталитический риформинг, обобщенный критерий оптимальности, нечеткая цель, нечеткое ограничение, нечеткое решение.

\section{Введение}

В настоящее время актуальным является направление повышения эффективности технологических процессов на основе разработки автоматизированных систем управления [1, 2]. Большое значение для экономического развития России имеют технологические процессы и производства нефтеперерабатывающей и нефтехимической промышленности. Одним из важнейших процессов нефтепереработки и нефтехимии является каталитический риформинг (КР), основное назначение которого состоит в получении высокооктанового бензинового топлива [3]. Существенный вклад в разработку и совершенствование подходов к моделированию и управлению процессом КР внесли такие ученые, как В.В. Кафаров [4], И.Н. Дорохов [4], Г.М. Панченков [5], Н.Ф. Рубекин [6], Ю.М. ЖЖоров [7], А.В. Кравцов [8], Э.Д. Иванчина [8], А.Г. Шумихин [9] и др.
Существующие системы управления процессом КР реализуют: оптимальное распределение температур на входах в реакторы [10], оптимизацию технологического режима процесса [11], управление качеством стабильного катализата [12], ситуационное управление процессом [13] и пр. При этом отсутствуют системы управления процессом КР, направленные на снижение издержек организации процесса и повышение октанового числа бензина.

Поскольку эффективность производства бензина зависит не только от повышения октанового числа выпускаемого топлива, но и от снижения издержек организации процесса, является актуальной задача, связанная с разработкой системы управления процессом КР, обеспечивающей достижение оптимальных соотношений октанового числа бензина и издержек организации процесса. Для исследования процесса КР необходимо провести его анализ как объекта управления. 


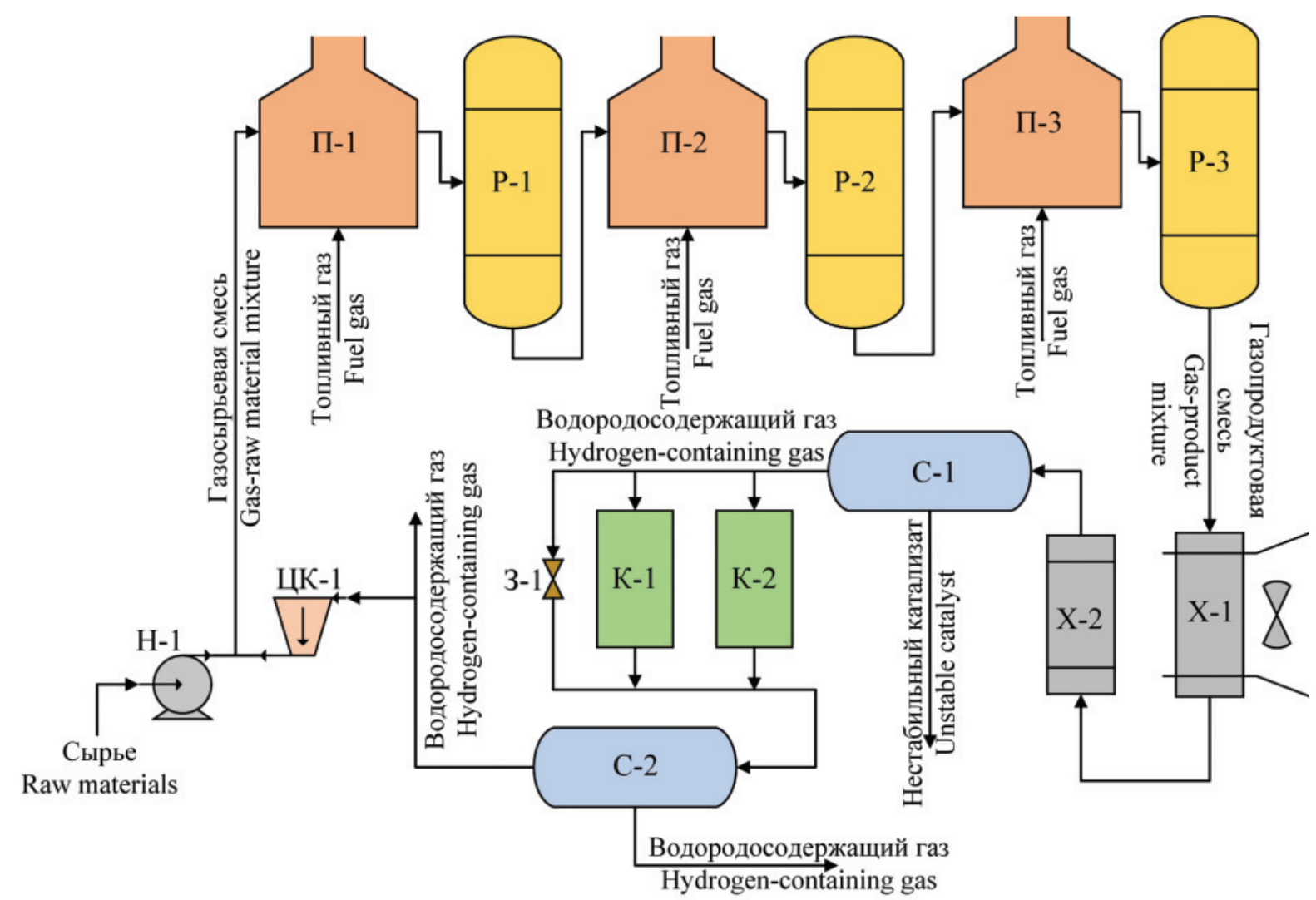

Pис. 1. Упрощенная технологическая схема процесса каталитического риформинга

Fig. 1. Catalytic reforming simplified flow chart

\section{Постановка задачи управления}

Основными аппаратами процесса КР являются ректоры P-1, Р-2, Р-3 и печи П-1, П-2, П-3, к вспомогательным аппаратам относятся: аппарат воздушного охлаждения $\mathrm{X}-1$, водяной холодильник $\mathrm{X}-2$, сепараторы C-1, C-2, адсорберы-осушители К-1, К-2, центробежный компрессор ЦК-1, насос H-1 (рис. 1) [14].

Процесс КР как объект управления характеризуется набором входных и выходных параметров (рис. 2). Все параметры были разделены на четыре группы и представлены в векторной форме:

1. Вектор управляющих воздействий $\mathrm{U}$ : расход топливного газа $Q_{f g} ;$ расход сырья $Q_{r}$.

2. Вектор возмущающих воздействий $\mathbf{V}$ : качество сырья $Q R$; качество топливного газа $Q F G$.

3. Вектор режимных параметров процесса $\mathbf{A}$ : температура на входе $T_{\text {in }}$ и выходе $T_{\text {out }}$ печи; давление в реакторе $P$; перепад температуры в реактоpe $\Delta T$; расход водородосодержащего газа $Q_{h g}$; активность катализатора $A C$; состояние печи $C F$.

4. Вектор выходных параметров $\mathbf{W}$ : октановое число бензина $\mathrm{ON}$; издержки организации процесса $Z[15]$.

Особенностью объекта является наличие качественной информации, входящей в состав векторов режимных параметров процесса $\mathbf{A}$ и возмуща- ющих воздействий $\mathbf{F}$ : активность катализатора $A C$ (низкая N, ниже средней ZN, средняя Z, выше средней ZР, высокая Р); качество сырья $Q R$ (низкое N, ниже среднего ZN, среднее Z, выше среднего ZP, высокое Р); качество топливного газа $Q F G$ (низкое N, ниже среднего ZN, среднее Z, выше среднего ZP, высокое P); состояние печи $C F$ (низкое N, ниже среднего ZN, среднее Z, выше среднего ZР, высокое Р). В работе [16] обоснована целесообразность учета данных параметров в математической модели (MМ) процесса КР как лингвистических переменных (ЛП), с использованием которых предложено учитывать накопленный опыт операторов-технологов. Данные переменные не могут быть измерены обычными способами и вводятся оператором. Предложенная модель применяется в системе управления при поиске экстремума целевой функции. Формализация качественной информации производится на основе методов экспертного опроса. На основе данного подхода определены функции принадлежности (ФП) для каждой ЛП процесса КР. Функции принадлежности могут уточняться с изменением экспертного мнения.

Цель управления - достижение минимального значения обобщенного критерия оптимальности (ОКО) (1) в условиях переменного качества сырья и качества топливного газа. 


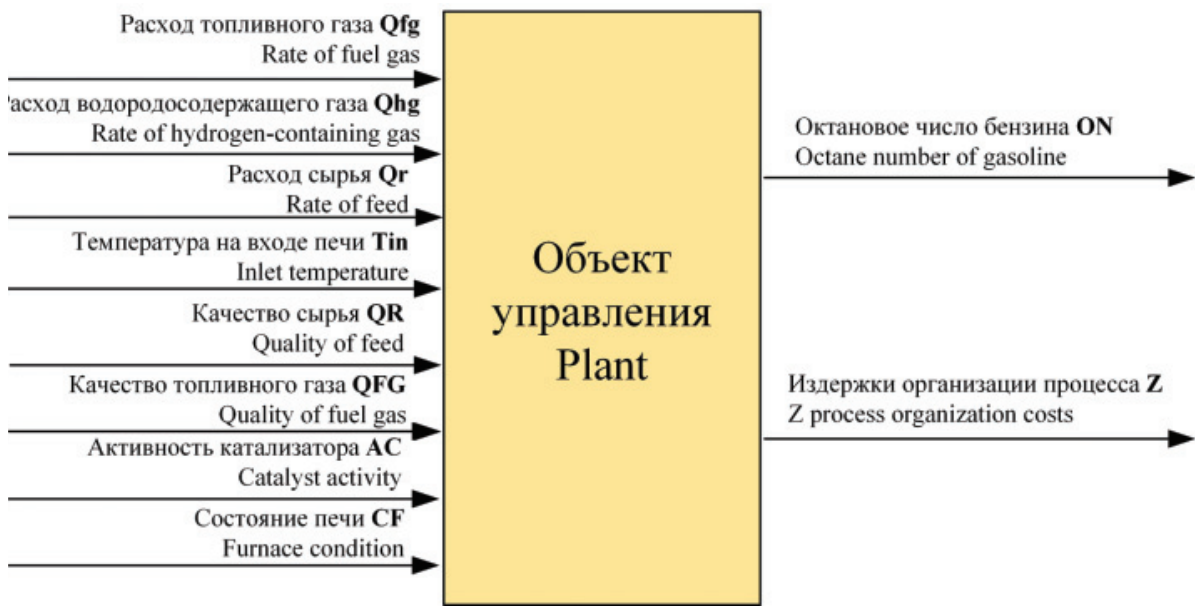

Рис. 2. Наборы входных и выходных паралетров процесса как объекта управления

Fig. 2. Sets of input and output parameters of the process as a control object

$$
J=k_{1} O N_{0} \frac{1}{O N}+\frac{k_{2}}{Z_{0}} Z=k_{1} J_{1}+k_{2} J_{2},
$$

где $J$ - OКо; $O N_{0}$ - минимальное значение октанового числа бензина; $Z_{0}$ - максимальные издержки организации процесса; $J_{1}$ - нормированный критерий минимума величины, обратной октановому числу бензина $O N ; J_{2}$ - нормированный критерий минимума издержек организации процесса $Z$; $k_{1}, k_{2}$ - весовые коэффициенты, регулирующие относительную важность критериев $J_{1}, J_{2}$ в отношении принятия решений, $k_{1}+k_{2}=1,0<k_{1}<1,0<k_{2}<1$.

Выбор критерия (1) обусловлен необходимостью объединения частных критериев и приведения их к безразмерной величине.

Задача управления процессом КР мощностью 1 млн т/год по сырью (2) формулируется следующим образом: найти оптимальные решения по управлению процессом КР, при которых достигается минимум ОКО с учетом воздействия возмущений $\mathbf{V}$ и заданных ограничений на выходные параметры W:

$$
\begin{gathered}
F\left(\begin{array}{c}
Q_{f g}, Q_{h g}, Q_{r}, T_{\text {in }}, \\
Q R, Q F G, A C, C F
\end{array}\right)=\min _{\left\{Q_{r}, Q_{f g}\right\} \in U} J, \\
\left\{Q_{r}, Q_{f g}\right\} \in U ; \\
\left\{Q_{h g}, T_{\text {in }}, T_{\text {out }}, \Delta T, P, A C, C F\right\} \in A ; \\
{[Q R, Q F G] \in V ;} \\
\{O N, Z\} \in W ;
\end{gathered}
$$

при ограничениях (3):

$$
O N \geq 92
$$

$$
\begin{gathered}
Z \leq 7,6 \text { (млн руб.); } \\
\underline{U_{i}} \leq U_{i} \leq \overline{U_{i}}, i=\overline{1, n ;} \\
\underline{A_{j}} \leq A_{j} \leq \overline{A_{j}}, j=\overline{1, m ;} \\
\underline{V_{l}} \leq V_{l} \leq \overline{V_{l}}, l=\overline{1, p ;} \\
\underline{W_{k}} \leq W_{k} \leq \overline{W_{k}}, k=\overline{1, q},
\end{gathered}
$$

где $\left\{Q_{r}, Q_{f g}\right\}$ - решения по управлению; $\mathbf{U}$ - вектор управлений; A - вектор режимных параметров объекта; V - вектор возмущений; W - вектор выходных параметров.

Анализ современных задач управления процессом КР показал, что отсутствует общий подход к решению задачи, решения существуют только по отдельным направлениям и применяемые в них методы управления не являются достаточно әффективными в условиях воздействия возмущений (качества сырья, качества топливного газа) [17].

На основании выявленных недостатков сделан вывод о том, что главная причина сложившейся ситуации в современной теории и практике управления процессом КР заключается в отсутствии системного анализа связей между выходными величинами и возмущающими воздействиями.

Результаты анализа показали, что усовершенствование управления процессом КР должно заключаться в использовании системой управления:

- расчетных и экспериментальных значений выходных параметров;

- качественной информации о возмущениях;

- управляющих воздействий, определенных по ММ процесса;

- нечетких целей и ограничений для учета экспертной информации при определении оптимальных решений по управлению процессом.

Сущность предлагаемого решения заключается в компенсации системой управления возмущений за счет учета экспертной информации об объекте и достижения оптимальных соотношений октанового числа бензина и издержек организации процесса.

Для достижения цели управления предлагается концепция управления процессом КР, состоящая в том, что оптимизация процесса производится с учетом экспертной информации о действующих возмущениях, причем начальной точкой поиска являются оптимальные значения, найденные без учета возмущений. 
Алгоритл поиска начальной точки оптилиза иии проиесса КР представим в виде последовательности решений по управлению процессом КP $U$, при которых достигается минимум ОКО без учета воздействия возмущений $\mathbf{V}$.

Для решения задачи условной оптимизации (2), относящейся к классу задач нелинейного программирования, предложено использование метода штрафных функций. При этом исходная задача условной оптимизации сводится к задаче безусловной оптимизации [18].

Среди существующих типов штрафов выбран штраф типа «бесконечный барьер», отличающийся простотой своего вычисления. Приведем описание функции штрафа для управляющих воздействий (4):

$$
\begin{gathered}
\Omega\left(Q_{r}\right)=\left\{\begin{array}{cc}
0, \text { если } & Q_{r} \geq 190 ; \\
10^{5}, \text { если } & Q_{r} \leq 130 ;
\end{array}\right. \\
\Omega\left(Q_{f g}\right)=\left\{\begin{array}{cc}
0, \text { если } & Q_{f g} \geq 1150 ; \\
10^{5}, \text { если } & Q_{f g} \leq 750 .
\end{array}\right.
\end{gathered}
$$

Для поиска экстремума целевой функции $J(x, y)$, где $x$ - расход сырья, $y$ - расход топливного газа, рассматривались методы прямого поиска, не требующие вычисления производных, поскольку целевая функция в настоящей работе не является дифференцируемой. При этом выбран метод конфигураций, достоинствами которого перед другими методами безусловной оптимизации (МонтеКарло, Нелдера-Мида, Розенброка, сопряженных направлений, поиска по симплексу и т. д.) являются простота стратегии поиска и небольшой объем требуемой памяти [19].

На основе метода конфигураций и метода штрафных функций был разработан алгоритм поиска начальной точки оптимизации процесса КР. C использованием данного алгоритма были получены результаты поиска решений по управлению процессом КР $U$ : расход сырья $x_{0}=160\left(\mathrm{M}^{3} /\right.$ ч) и расход топливного газа $y_{0}=950\left(\mathrm{M}^{3} /\right.$ ч).

Алгоритлол оптилизации процесса КР с учетол экспертной инфорлации является процедура поиска оптимальных решений по управлению процессом КР $U^{*}$, при которых достигается минимум ОКО с учетом воздействия возмущений $\mathbf{V}$.

Для учета воздействия возмущений при поиске оптимальных решений по управлению процессом КР предложено использование схемы Беллмана-Заде. Данный подход позволяет определять оптимальные решения $U^{*}$, используя результаты поиска решений по управлению процессом $U$ на основе задания нечетких целей и ограничений и определения нечеткого решения. Иллюстрация данного подхода применительно к поиску оптимального расхода сырья приведена на рис. 3 [20].

На рис. 3 показано пересечение функций принадлежности (ФП) нечетких множеств, соответствующих нечеткой цели и двум нечетким ограничениям.
Нечеткая цель $G$ задана в виде высказывания «значение ОКО должно быть минимально», отражает главную цель управления процессом КР и представлена в виде гауссовой ФП с точкой максимума, соответствующей решению по управлению процессом $U=\left\{x_{0}, y_{0}\right\}$, при котором достигается минимум ОКО без учета воздействия возмущений $\mathbf{V}$.

Первое нечеткое ограничение $C_{1}$ задано в виде высказывания «активность катализатора должна быть выше средней», отражает необходимое требование к режиму процесса КР и представлено в виде сигмоидной ФП, точка перегиба которой соответствует решению по управлению процессом $U_{1}=\left\{x_{1}, y_{1}\right\}$, рекомендованному лицом, принимающим решение (ЛПР), для компенсации возмущения: изменения качества сырья $Q R$.

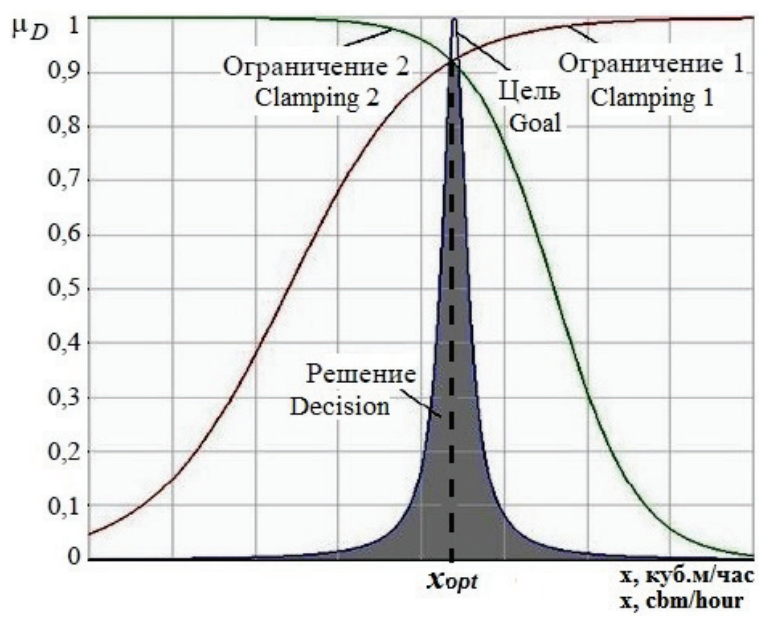

Рис.3. Поиск оптимального расхода сырья по схеме Беллма$н a-3 a \partial e$

Fig. 3. Search for the optimal consumption of raw materials according to the Bellman-Zade scheme

Второе нечеткое ограничение $C_{2}$ задано в виде высказывания «состояние печи риформинга должно быть лучше среднего», отражает необходимое требование к режиму процесса КР и представлено в виде сигмоидной ФП, точка перегиба которой соответствует решению по управлению процессом $U_{2}=\left\{x_{2}, y_{2}\right\}$, рекомендованному ЛПР для компенсации возмущения: изменения качества топливного газа $Q F G$.

На основе конфликта цели и ограничений получаем область значений ФП $\mu_{D}(x, y)$ «Решение», соответствующую множеству оптимальных решений по управлению процессом КР. Из данной области определяем координаты $\left(x_{\text {opt }} ; y_{\text {opt }}\right)$, соответствующие максимуму ФП нечеткого решения (5).

$$
\begin{gathered}
\mu_{D}\left(x_{o p t}, y_{o p t}\right)=\max \mu_{D}(x, y)= \\
=\max \min \left(\mu_{G}(x, y), \mu_{C_{1}}(x, y), \mu_{C_{2}}(x, y)\right) .
\end{gathered}
$$

где $\mu_{D}(x, y)$ - гауссова ФП, соответствующая нечеткой цели; $\mu_{c 1}(x, y)$ - сигмоидная $Ф П$, соответствующая первому нечеткому ограничению; $\mu_{c 2}(x, y)-$ сигмоидная ФП, соответствующая второму нечеткому ограничению. 


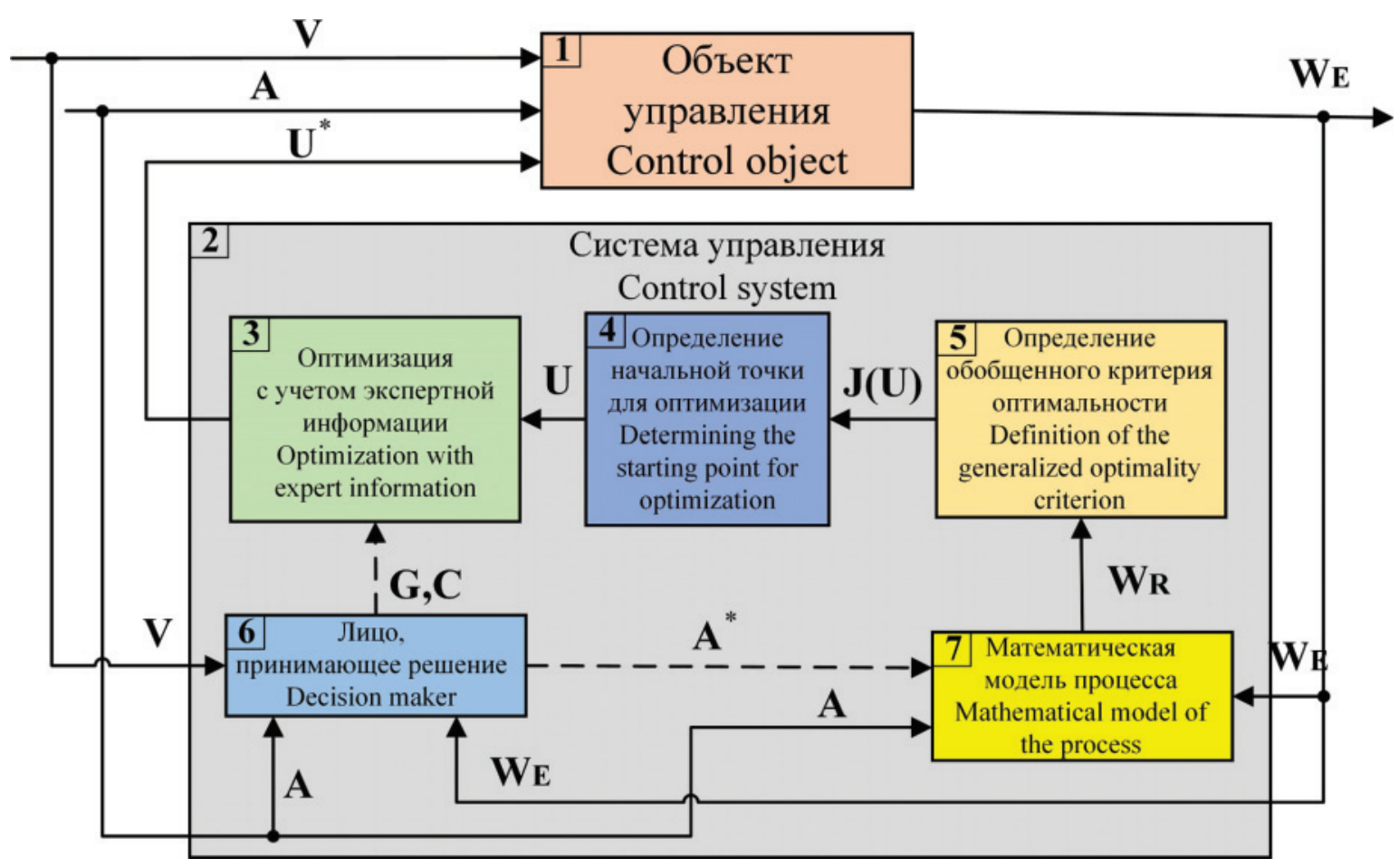

Рис. 4. Структура системы управления процессом каталитического рифорлинга

Fig. 4. Structure of the catalytic reforming process control system

На основе схемы Беллмана-Заде был разработан алгоритм оптимизации процесса КР с учетом экспертной информации. С использованием данного алгоритма были получены результаты поиска оптимальных решений по управлению процессом КР $U^{*}$ : расход сырья $x_{\text {орt }}=162\left(\mathrm{~m}^{3} /\right.$ ч) и расход топливного газа $y_{\text {opt }}=970\left(\mathrm{M}^{3} /\right.$ ч).

\section{Реализация и полученные результаты}

Для решения поставленной задачи разработано программное обеспечение на языке Visual C\#. На рис. 4 представлена структура системы управления процессом КР.

Система управления процессом КР - 2 включает ряд блоков: блок определения ОКО по ММ процесса -5 , блок определения начальной точки для оптимизации -4 , блок оптимизации с учетом экспертной информации - 3.

На вход ММ процесса КР - 7 поступают значения вектора режимных параметров А в виде данных контрольно-измерительных приборов, экспериментальные значения вектора выходных пере-

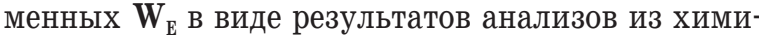
ко-аналитической лаборатории, а также ЛПР - 6 задает значения качественных режимных параметров $\mathbf{A}^{*}$ (активность катализатора, состояние печи риформинга).

На вход блока - 5 поступают расчетные значения вектора выходных переменных $\mathbf{W}_{R}$, полученные на выходе ММ процесса КР - 7.

На основе блока - 4 определяются решения по управлению $U$, соответствующие минимуму ОКО $J$
$(U)$ без учета воздействия возмущений $\mathbf{V}$. Полученные решения по управлению необходимы для задания нечеткой цели в блоке -3.

В блоке - 3 определяются оптимальные решения по управлению $U^{*}$, соответствующие минимуму ОКО $J(U)$ с учетом воздействия возмущений $\mathbf{V}$, которые в качестве управляющий воздействий поступают на объект управления - 1 .

Выходные параметры процесса КР, октановое число бензина и издержки организации процесса находятся в состоянии антагонизма, т. е. улучшение одного из них приводит к ухудшению другого.

Результаты сравнения АСУ процесса КР по среднесуточным издержкам организации процесса показали, что при управлении процессом на основе разработанной системы, примененной в течение месяца работы установки Л-35-11/1000, обеспечивается снижение среднесуточных издержек организации процесса на 0,24 млн р. по сравнению с управлением процессом по проектному решению, примененному за аналогичный период времени. C учетом допущения, что одинаков характер протекания процесса КР на установке Л-35-11/1000 в течение года, определено снижение среднегодовых издержек организации процесса при управлении процессом на основе разработанной системы, равное 86,74 млн р. При сравнении АСУ процесса КР по среднему октановому числу были вычислены средние значения данного параметра: 92,37 - при управлении процессом по проектному решению, примененному в течение месяца работы установки Л-35-11/1000; 93,47 - за аналогичный период вре- 


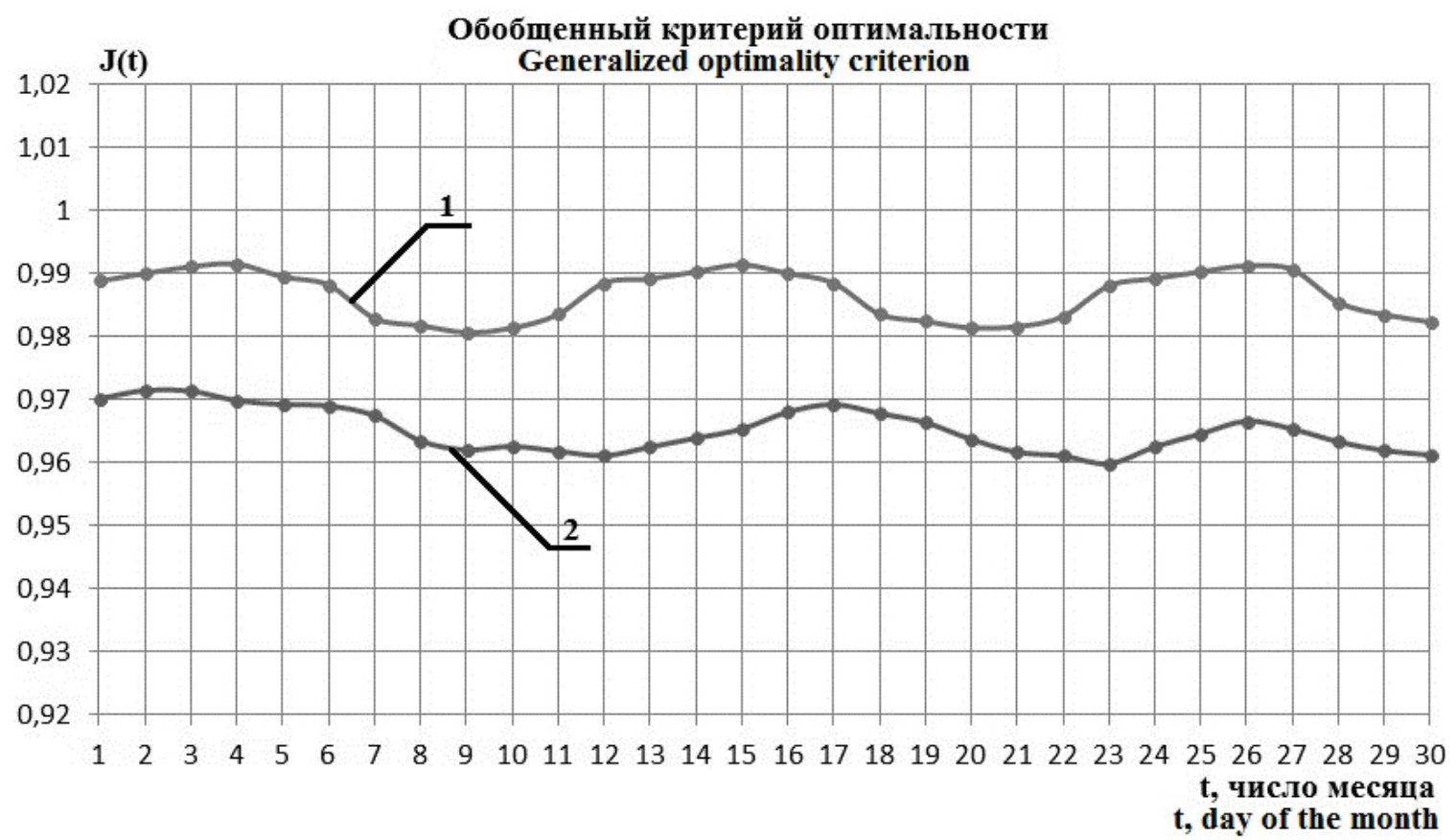

Pис. 5. Изменение обобщенного критерия оптимальности

Fig. 5. Modification of the generalized optimality criterion

мени, но при управлении на основе разработанной системы. Исходя из этого было установлено, что при управлении процессом на основе разработанной системы, примененной в течение месяца работы установки Л-35-11/1000, обеспечивается повышение среднего октанового числа бензина на 1,1 по сравнению с управлением процессом по проектному решению, примененному за аналогичный период времени. Разработанная система управления обеспечивает уменьшение среднегодовых издержек организации процесса на 86,74 млн р. при отсутствии увеличения октанового числа, а также обеспечивает повышение среднего октанового числа бензина на 1,1 при отсутствии уменьшения издержек организации процесса.

На рис. 5 приведен пример ожидаемого снижения ОКО: 1 - при управлении процессом по проект-

\section{СПИСОК ЛИТЕРАТУРЫ}

1. Программно-алгоритмический комплекс защиты и управления предприятием / Б.С. Дмитриевский, В.Г. Матвейкин, В.И. Медников, С.Г. Семержинский // Программные продукты и системы. - 2017. - № 2. - С. 307-313.

2. Управление инновационным развитием наукоемкой производственной системы / В.Г. Матвейкин, Б.С. Дмитриевский, О.В. Дмитриева, В.Р. Разиева, М.В. Сивова // Вопросы современной науки и практики. - 2016. - № 3 (61). - С. 146-152.

3. Catalytic Cracking (FCC) Process Modeling, Simulation, and Control / C.I.C. Pinheiro, J.L. Fernandes, L. Domingues et al. // Industrial I Engineering Chemistry Research. -2012. - № 51 (1). P. 1-29.

4. Кафаров В.В., Дорохов И.Н. Системный анализ процессов химической технологии. Основы стратегии. - М.: Наука, 1976. $500 \mathrm{c.}$ ному решению, примененному в течение месяца работы установки Л-35-11/1000, 2 - за аналогичный период времени, но при управлении на основе разработанной системы. Среднее значение ОКО уменьшилось на 2,2 \% .

\section{Заключение}

Таким образом, проведен анализ существующих подходов к управлению процессом каталитического риформинга; разработана система управления процессом каталитического риформинга, включающая алгоритм поиска начальной точки оптимизации процесса и алгоритм оптимизации процесса с учетом экспертной информации; поставлена и решена задача управления процессом каталитического риформинга по обобщенному критерию оптимальности при ограничениях на выходные параметры.

5. Панченков Г.М. Каталитические и радиационно-химические процессы нефтепереработки и нефтехимии. - М.: Химия, 1982. $-152 \mathrm{c}$.

6. Рубекин Н.Ф. Системы автоматического оптимального управления каталитическими процессами платформинга и гидроочистки. - М.: ЦНИИТЭНЕФТЕХИМ, 1972. - 66 с.

7. Жоров Ю.М. Расчеты и исследования каталитических процессов нефтепереработки. - М.: Химия, 1973. - 216 с.

8. Математическое моделирование каталитических процессов переработки углеводородного сырья / А.В. Кравцов, Э.Д. Иванчина, Е.Н. Ивашкина, А.В. Костенко, Е.М. Юрьев, В.С. Бесков // Катализ в промышленности. - 2008. - № 6. C. $41-46$.

9. Применение многоуровневой математической модели процесса каталитического риформинга бензиновых фракций в системе управления промышленными установками / В.Г. Плехов, 
С.Н. Кондрашов, А.Г. Шумихин // Автоматизация в промышленности. - 2009. - № 7. - С. 37-42.

10. Coleman B., Babu J. Techniques of Model-Based Control. - Upper Saddle River: Pretice Hall PTTr, 2002. - 576 p.

11. Bequette W. Process Control Modeling Design and Simulation. Upper Saddle River: Pretice Hall PTTr, 2003. - 564 p.

12. Gumen M.I. et al. Increasing of the Efficiency of the Reforming LG-35-11/300 // Petroleum Processing and Petrochemistry. 2001. - № 11. - P. 54-57.

13. Mircea C., Agachi S., Marimoiu V. Simulation and Model Predictive Control of a UOP Fluid Catalytic Cracking // Chemical Engineering and Processing. -2003 . - V. 42. - P. 67.

14. Ancheyta J. Modeling and simulation of catalytic reactors for petroleum refining. - Hoboken: Wiley, 2011. $-528 \mathrm{p}$.

15. Weekman V.A. Model of Catalytic Cracking Conversion in Fixed, Moving and Fluid-Bed Reactors // Industrial and Engineering Chemistry Process Design and Development. - 1968. - № 7 (1). P. 90-95.

16. Проталинский 0.М. Применение методов искусственного интеллекта при автоматизации технологических процессов: мо- нография. - Астрахань: Изд-во Астрах. гос. техн. ун-та, 2004. $-183 \mathrm{c}$.

17. Smith J.M. Chemical engineering kinetics. - St. Louis: McGraw-Hill, 1981. - $676 \mathrm{p}$.

18. Литовка Ю.В., Као В.З. Моделирование и оптимизация гальванической ванны с дополнительными катодами // Вестник Тамбовского государственного технического университета. 2016. - T. 22. - № 1. - C. 68-74.

19. Литовка Ю.В., Као В.З., Соловьёв Д.С. Оптимизация гальванической ванны с дополнительными катодами и биполярными электродами // Вестник Астраханского государственного технического университета. Серия: Управление, вычислительная техника и информатика. - 2016. - № 2. - С. 7-17.

20. Джамбеков А.М. Использование информационных технологий для обеспечения автоматизированного управления процессом каталитического риформинга в условиях неопределенности // Прикаспийский журнал: управление и высокие технологии. 2017. - № 2 (38). - C. 36-46.

Поступила 12.11.2018 2.

\section{Информация об авторах}

Матвейкин В.Г., доктор технических наук, профессор, заведующий кафедрой информационных процессов и управления Института автоматики и информационных технологий Тамбовского государственного технического университета.

Дмитриевский Б.С., доктор технических наук, доцент, профессор кафедры информационных процессов и управления Института автоматики и информационных технологий Тамбовского государственного технического университета.

Кокуев A.Г., кандидат технических наук, доцент, заведующий кафедрой автоматики и управления Института информационных технологий и коммуникаций Астраханского государственного технического университета.

Джалбеков A.M., аспирант кафедры автоматики и управления Института информационных технологий и коммуникаций Астраханского государственного технического университета. 
UDC 681.5

\title{
PROBLEM OF CONTROL OF CATALYTIC REFORMING AND METHOD OF ITS SOLUTIONS
}

\author{
Valery G. Matveykin',
}

ipu@ahp.tstu.ru

Boris S. Dmitrievsky',

dmiboris@yandex.ru

\section{Andrey G. Kokuev'} kokuevag@gmail.com

\author{
Azamat M. Dzhambekov', \\ azamat-121@mail.ru \\ 1 Tambov State Technical University, \\ 106, Sovetskaya Street, Tambov, 392000, Russia. \\ ${ }^{2}$ Astrakhan State Technical University, \\ 16, Tatishchev Street, Astrakhan, 414056, Russia.
}

The relevance of the research is caused by the need to ensure optimal ratios of the octane number of gasoline and the cost of organizing the process in a catalytic reformer with a capacity of 1 million tons per year for raw materials in conditions of variable quality of raw materials and quality of fuel gas. The effectiveness of gasoline production depends not only on increasing the octane number, but also on reducing the costs of the process organization. The existing systems for controlling the catalytic reforming do not ensure the achievement of optimal ratios of the octane number of gasoline and the costs of the process organization. It is necessary to develop a control system for the catalytic reforming process, which ensures the achievement of these ratios.

The main aim of the research is to achieve optimum octane number ratio of gasoline and process organization costs in a catalytic reformer with a capacity of 1 million tons per year for raw materials in conditions of varying quality of raw materials and quality of fuel gas by developing a management system.

Object of the research is the catalytic reforming unit with a capacity of 1 million tons per year for raw materials.

Methods: fuzzy sets theory, decision theory in fuzzy conditions, computational mathematics, automatic control theory.

Results. The authors have set the task of controlling the process of catalytic reforming at a L-35-11 / 1000 reformer with a capacity of 1 million tons per year for raw materials and carried out the analysis of the process as a control object with the identification of its features. The paper describes the set of input and output parameters of the control object and introduces the expression for determining the generalized optimality criterion. The authors developed the system for controlling the catalytic reforming. The implementation of the proposed system ensures the achievement of optimal ratios of the octane number of gasoline and production costs.

\section{Key words:}

Catalytic reforming, generalized optimality criterion, fuzzy goal, unclear constraint, fuzzy solution.

\section{REFERENCES}

1. Dmitrievsky B.S., Matveykin V.G., Mednikov V.I., Semerzhinsky S.G. Program-algorithmic complex of protection and enterprise management. Software products and systems, 2017, no. 1, pp. 307-313. In Rus.

2. Matveykin V.G., Dmitrievsky B.S., Dmitrieva O.V., Razieva V.R., Sivova M.V. Management of innovative development of a knowledge-based production system. Issues of modern science and practice, 2016, no. 3 (61), pp. 146-152. In Rus.

3. Pinheiro C.I.C., Fernandes J.L., Domingues L. Catalytic Cracking (FCC) Process Modeling, Simulation, and Control. Industrial I Engineering Chemistry Research, 2012, no. 51 (1), pp. 1-29.

4. Kafarov V.V., Dorokhov I.N. Sistemny analiz protsessov khimicheskoy tekhnologii. Osnovy strategii [System analysis of the processes of chemical technology. Fundamentals of strategy]. Moscow, Nauka Publ., 1976. 500 p.

5. Panchenkov G.M. Kataliticheskie i radiatsionno-khimicheskie processy neftepererabotki i neftekhimii [Catalytic and radiationchemical processes of oil refining and petrochemistry]. Moscow, Khimiya Publ., 1982. 152 p.

6. Rubekin N.F. Sistemy avtomaticheskogo optimalnogo upravleniya kataliticheskimi protsessami platforminga i gidroochistki [Systems of automatic optimal control of catalytic processes of plat- forming and hydrotreatment]. Moscow, TsNIITENEFTEHIM Publ., $1972.66 \mathrm{p}$.

7. Zhorov Yu.M. Raschety i issledovaniya kataliticheskikh protsessou neftepererabotki [Calculations and studies of catalytic processes of oil refining]. Moscow, Khimiya Publ., 1973. 216 p.

8. Kravtsov A.V., Ivanchina E.D., Ivashkina E.N., Kostenko A.V., Yuryev E.M., Beskov V.S. Mathematical modeling of catalytic processes of hydrocarbon feedstock processing. Catalysis in the industry, 2008, no. 6, pp. 41-46. In Rus.

9. Plekhov V.G., Kondrashov S.N., Shumikhin A.G. Application of a multilevel mathematical model of the process of catalytic reforming of gasoline fractions in the industrial plant control system. Automation in the industry, 2009, no. 7, pp. 37-42. In Rus.

10. Coleman B., Babu J. Techniques of Model-Based Control. Upper Saddle River, Prentice Hall PTr Publ., 2002. 576 p.

11. Bequette W. Process Control Modeling Design and Simulation. Upper Saddle River, Pretice Hall PTTr Publ., 2003. 564 p.

12. Gumen M.I. Increasing of the Efficiency of the Reforming LG-35-11/300. Petroleum Processing and Petrochemistry, 2001, no. 11 , pp. 54-57.

13. Mircea C., Agachi S., Marimoiu V. Simulation and Model Predictive Control of a UOP Fluid Catalytic Cracking. Chemical Engineering and Processing, 2003, vol. 42, p. 67. 
14. Ancheyta J. Modeling and simulation of catalytic reactors for pet roleum refining. Hoboken, Wiley Publ., 2011. 528 p.

15. Weekman V.A. Model of Catalytic Cracking Conversion in Fixed, Moving and Fluid-Bed Reactors. Industrial and Engineering Chemistry Process Design and Development, 1968, no. 7 (1), pp. $90-95$.

16. Protalinsky 0.M. Primenenie metodov iskusstvennogo intellekta pri avtomatizatsii tekhnologicheskikh protsessov: monografiya [Application of artificial intelligence methods in automation of technological processes: monograph]. Astrakhan, Astrakhan State Technical University Publ. house, 2004. 183 p.

17. Smith J.M. Chemical engineering kinetics. St. Louis, McGrawHill Publ., 1981. 676 p.
18. Litovka Yu.V., Kao V.Z. Modeling and optimization of a galvanic bath with additional cathodes. Bulletin of the Tambov State Technical University, 2016, vol. 22, no. 1, pp. 68-74. In Rus.

19. Litovka Yu.V., Kao V.Z., Solovyov D.S. Optimization of galvanic bath with additional cathodes and bipolar electrodes. Bulletin of the Astrakhan State Technical University. Series: Management, Computer Science and Informatics, 2016, no. 2, pp. 7-17. In Rus.

20. Dzhambekov A.M. Use of information technologies to provide automated control over the process of catalytic reforming in conditions of uncertainty. Prikaspiysky Journal: Management and High Technologies, 2017, no. 2 (38), pp. 36-46. In Rus.

\section{Information about the authors}

Valery G. Matveykin, Dr. Sc., professor, Tambov State Technical University.

Boris S. Dmitrievsky, Dr. Sc., associate professor, Tambov State Technical University.

Andrey G. Kokuev, Cand. Sc., associate professor, Astrakhan State Technical University.

Azamat M. Dzhambekov, graduate student, Astrakhan State Technical University. 